Article

\title{
Some Results on Various Cancellative CA-Groupoids and Variant CA-Groupoids
}

\author{
Zhirou Ma ${ }^{1}$, Xiaohong Zhang ${ }^{1, *}$ and Florentin Smarandache ${ }^{2}$ (I) \\ 1 Department of Mathematics, Shaanxi University of Science \& Technology, Xi'an 710021, China; \\ 1809037@sust.edu.cn \\ 2 Department of Mathematics, University of New Mexico, Gallup, NM 87301, USA; smarand@unm.edu \\ * Correspondence: zhangxiaohong@sust.edu.cn or zhangxh@shmtu.edu.cn
}

Received: 19 January 2020; Accepted: 16 February 2020; Published: 22 February 2020

\begin{abstract}
Cyclic associativity can be regarded as a kind of variation symmetry, and cyclic associative groupoid (CA-groupoid) is a generalization of commutative semigroup. In this paper, the various cancellation properties of CA-groupoids, including cancellation, quasi-cancellation and power cancellation, are studied. The relationships among cancellative CA-groupoids, quasi-cancellative CA-groupoids and power cancellative CA-groupoids are found out. Moreover, the concept of variant CA-groupoid is proposed firstly, some examples are presented. It is shown that the structure of variant CA-groupoid is very interesting, and the construction methods and decomposition theorem of variant CA-groupoids are established.
\end{abstract}

Keywords: cyclic associative groupoid (CA-groupoid); cancellative; variant CA-groupoids; decomposition theorem; construction methods

\section{Introduction}

An algebraic structure is called a groupoid, if it is well-defined regarding an operation on it. A groupoid satisfying the "cyclic associative law" (that is, $x(y z)=y(z x)$ ) is called a cyclic associative groupoid, or simply CA-groupoid [1,2].

In fact, as early as 1946, when Byrne [3] studied axiomatization of Boolean algebra, he mentioned the following operation law: $(x y) z=(y z) x$. Obviously, its dual form is as follows: $z(y x)=x(z y)$, this is the cyclic associative law mentioned above. In 1954, Sholander [4] mentioned Byrne's paper [3], and used the term of "cyclic associative law" to express the operation law: $(a b) c=(b c) a$. This is the first literature we know to use the term "cyclic associative law". At the same time, Hosszu also used the term of "cyclic associative law" in the study of functional equation (see [5] and the introduction and explanation by Maksa [6]). Later, Kleinfeld [7] and Behn [8,9] studied the rings satisfying the cyclic associative law, and Iqbal et al. [10] studied the AG-groupoids satisfying the cyclic associative law. It is on the basis of these researches that we start to systematically study the groupoids satisfying the cyclic binding law (CA-groupoids) in [1,2], in order to provide a common basis for the research of related algebraic systems.

As a continuation of [1,2], this paper focuses on various cancellation properties of CA- groupoids and a special class of CA-groupoids. In many algebraic systems (such as semigroups, commutative semigroups and AG-groupoids), the cancellation, quasi-cancellation and power cancellation properties have important research value (see [11-26]). In 1957, Takayuki Tamura studied commutative non-potent Archimedean semigroups with cancellative law (see [11]), cancellability is applied to semigroups. Since then, various cancellative laws have been put forward and applied to various algebraic systems, and a series of valuable conclusions have been drawn. The rise of these properties makes an irreplaceable contribution to the development of algebra. 
Semigroup with the identity is named monoid, the research of monoid is gradually deepening (see [24,27]). In addition, AG-group is an AG-groupoid with the left identity and inverses (see [28-32]). Through these papers, we know that the identity is a powerful tool for solving algebraic problems. Therefore, we naturally consider CA-groupoids with unit element. However, our study finds that CA-groupoids with unit element degenerate into commutative monoids, and a CA-groupoid with quasi right unit element (i.e., there exists $e$, if $x \neq e$, then $x e=x$; and $e e \neq e$ ) maybe not a semigroup. Moreover, this kind of CA-groupoids (with quasi right unit element) not only has very interesting properties, but also promotes the study of algebraic structures such as rings and semirings (some examples are presented in Section 5). Therefore, this paper studies it in depth, and we call them variant CA-groupoids.

At last, the content of this paper as follows: in Section 2, we introduce some basic concepts and cancellative properties on semigroup and AG-groupoid; in Section 3, we give the definitions of cancellative CA-groupoids, left cancellative CA-groupoids, right cancellative CA-groupoids and weak cancellative CA-groupoids, and discuss the relationships about them; in Section 4, we give the definitions of several quasi-cancellative CA-groupoids and power cancellative CA-groupoids, and analyze the relationships about several types cancellative CA-groupoids; in Section 5, we propose the new notion of variant CA-groupoid and some interesting examples, moreover, we prove the structure theorem and construction method of variant CA-groupoids.

\section{Preliminaries}

This paper mainly studies some special types of CA-groupoids. In this section some notions and results on semigroups and CA-groupoids are given. A groupoid $\left(S,{ }^{*}\right)$ is a non-empty set $S$ together with a binary operation *. Traditionally, the * operator is omitted without confusion, and $\left(S,{ }^{*}\right)$ is abbreviated to $S$. For a groupoid $S$, an element $a \in S$ is called to be left cancellative (respectively right cancellative) if for all $x, y \in S, a x=a y$ implies $x=y(x a=y a$ implies $x=y)$; an element is called to be cancellative if it is both left and right cancellative. A groupoid $S$ satisfying the associative law is called a semigroup. A monoid $S$ is a semigroup with an identity element.

Definition 1. [1] Let $S$ be a groupoid. If for all $a, b, c \in S, a(b c)=c(a b)$, then $S$ is called a cyclic associative groupoid (or shortly CA-groupoid).

Proposition 1. [1] If $S$ is a CA-groupoid, then, for any $a, b, c, d, x, y \in S$ :

(1) $(a b)(c d)=(d a)(c b)$;

(2) $(a b)((c d)(x y))=(d a)((c b)(x y))$.

Proposition 2. [1] Every commutative semigroup is a CA-groupoid. Assume that $(S, \cdot)$ is a CA-groupoid, if S is commutative, then $S$ is a commutative semigroup.

Proposition 3. [1] Let $S$ be a CA-groupoid. (1) If $S$ have a left identity element, that is, there exists e $\in S$ such that ea $=$ a for all $a \in S$, then $S$ is a commutative semigroup (thus, $S$ is a commutative monoid). (2) If e $\in S$ is a left identity element in $S$, then $e$ is an identity element in $S$. (3) If $e \in S$ is a right identity element in $S$, that is, ae =a for all $a \in S$, then $e$ is an identity element in $S$. (4) If $S$ have a right identity element, then $S$ is a commutative semigroup (thus, $S$ is a commutative monoid).

Proposition 4. [1] Let $S$ be a CA-groupoid. If for all $a \in S, a^{2}=a$, then $S$ is commutative (thus, $S$ is a commutative semigroup).

Proposition 5. [1] Let $S_{1}, S_{2}$ be two CA-groupoids. Then the direct product $S_{1} \times S_{2}$ is a CA-groupoid. 
Definition 2. [2] An element a of a CA-groupoid $S$ is called locally associative if satisfied:

$$
a(a a)=(a a) a .
$$

$S$ is called a locally associative CA-groupoid, if all elements in $S$ are locally associative.

Definition 3. [2] Let $S$ be a groupoid. If for all $a, b, c \in S$ :

$$
a(b c)=(a b) c, a(b c)=c(a b),
$$

then $S$ is called a cyclic associative semigroup (shortly, CA-semigroup).

Definition 4. [18] Let $S$ be a semigroup. $S$ is called a separative semigroup, if for any $x, y \in S$ :

(i) $x^{2}=x y$ and $y^{2}=y x$ imply $x=y$;

(ii) $x^{2}=y x$ and $y^{2}=x y$ imply $x=y$.

A semigroup $S$ is called quasi-separative if for all $a, b \in S, x^{2}=x y=y^{2}$ imply $x=y$.

\section{Cancellation Properties of CA-Groupoids}

Definition 5. Assume that $S$ is a CA-groupoid. If every element of $S$ is left cancellative (right cancellative, cancellative), then $S$ is called a left cancellative (right cancellative, cancellative) CA-groupoid.

Example 1. Let $S=\{\overline{0}, \overline{1}, \overline{2}, \overline{3}, \overline{4}\}$. For all $x, y \in S$, the operation * on $S$ is defined as $x^{*} y=\overline{x+y}=x+y$ (mod 5), see Table 1. Then, $\left(S,{ }^{*}\right)$ is a cancellative CA-groupoid.

Table 1. The operation * on $S$.

\begin{tabular}{cccccc}
\hline$*$ & $\overline{\mathbf{0}}$ & $\overline{\mathbf{1}}$ & $\overline{\mathbf{2}}$ & $\overline{\mathbf{3}}$ & $\overline{\mathbf{4}}$ \\
\hline$\overline{\mathbf{0}}$ & $\overline{0}$ & $\overline{1}$ & $\overline{2}$ & $\overline{3}$ & $\overline{4}$ \\
$\overline{\mathbf{1}}$ & $\overline{1}$ & $\overline{2}$ & $\overline{3}$ & $\overline{4}$ & $\overline{0}$ \\
$\overline{\mathbf{2}}$ & $\overline{2}$ & $\overline{3}$ & $\overline{4}$ & $\overline{0}$ & $\overline{1}$ \\
$\overline{\mathbf{3}}$ & $\overline{3}$ & $\overline{4}$ & $\overline{0}$ & $\overline{1}$ & $\overline{2}$ \\
$\overline{\mathbf{4}}$ & $\overline{4}$ & $\overline{0}$ & $\overline{1}$ & $\overline{2}$ & $\overline{3}$ \\
\hline
\end{tabular}

Definition 6. Assume that $S$ is a $C A$-groupoid. Let $x \in S$, if for any $y, z \in S, x y=x z$ and $y x=z x$ imply $y=z$, then $x$ is called to be weak cancellative. If all elements in $S$ are weak cancellative, then $S$ is called a weak cancellative CA-groupoid.

Obviously, for a CA-groupoid $S$ and any $x \in S$, if $x$ is a left (or right) cancellative, then $x$ is weak cancellative.

Example 2. Let $S=\{1,2,3,4\}$. The operation * on $S$ is defined as Table 2. Then, $\left(S,{ }^{*}\right)$ is a weak cancellative CA-groupoid.

Table 2. The operation * on $S$.

\begin{tabular}{lllll}
\hline$*$ & $\mathbf{1}$ & $\mathbf{2}$ & $\mathbf{3}$ & $\mathbf{4}$ \\
\hline $\mathbf{1}$ & 4 & 3 & 2 & 1 \\
$\mathbf{2}$ & 3 & 1 & 4 & 2 \\
$\mathbf{3}$ & 2 & 4 & 1 & 3 \\
$\mathbf{4}$ & 1 & 2 & 3 & 4 \\
\hline
\end{tabular}


Theorem 1. Let $S$ be a $C A$-groupoid. Then, for any element $a, b \in S$ :

(1) if a is left cancellative, then a is right cancellative, thus a is cancellative;

(2) if $a$ and $b$ are left cancellative, then $a b$ is right cancellative;

(3) if $a$ is right cancellative and $b$ is left cancellative, then ab is right cancellative;

(4) if $a b$ is right cancellative, then $a b=b a$;

(5) if ab is cancellative, then $b$ is cancellative;

(6) if $a b$ is cancellative, then $a$ and $b$ are cancellative;

(7) if $a$ and $a b$ are right cancellative, and $b$ is left cancellative, then $a$ is cancellative;

(8) if $a$ and $a b$ are right cancellative, and $b$ is left cancellative, then $a b$ is cancellative.

Proof. Suppose that $\left(S,{ }^{*}\right)$ is a CA-groupoid and $a, b \in S$.

(1) Assume that $a$ is a left cancellative element. If $(\forall x, y \in S) x^{*} a=y^{*} a$, then (by cyclic association):

$$
a^{*}\left(a^{*} x\right)=x^{*}\left(a^{*} a\right)=a^{*}\left(x^{*} a\right)=a^{*}\left(y^{*} a\right)=a^{*}\left(a^{*} y\right) .
$$

From this, applying left cancellation property of $a, a^{*} x=a^{*} y$. From this, applying left cancellation property of $a$ one time, we get that $x=y$. Therefore, $a$ is a right cancellative element in $S$, so $a$ is a cancellative element in $S$.

(2) Suppose that $a$ and $b$ are left cancellative. If $(\forall x, y \in S) x^{*} a b=y^{*} a b$, then:

$$
a^{*}\left(b^{*} x\right)=x^{*}\left(a^{*} b\right)=x^{*}(a b)=y^{*}(a b)=y^{*}\left(a^{*} b\right)=b^{*}\left(y^{*} a\right)=a^{*}\left(b^{*} y\right) .
$$

Since $a$ is left cancellative, so $b^{*} x=b^{*} y$. Moreover, from this and $b$ is left cancellative, we get that $x=y$. Therefore, $a b$ is a right cancellative.

(3) Assume that $a$ is right cancellative and $b$ is left cancellative. If $(\forall x, y \in S) x^{*} a b=y^{*} a b$, then:

$$
b^{*}\left(x^{*} a\right)=a^{*}\left(b^{*} x\right)=x^{*}\left(a^{*} b\right)=x^{*}(a b)=y^{*}(a b)=y^{*}\left(a^{*} b\right)=b^{*}\left(y^{*} a\right) .
$$

Since $b$ is left cancellative, so $x^{*} a=y^{*} a$. Moreover, from this and $a$ is right cancellative, we get that $x=y$. Therefore, $a b$ is a right cancellative.

(4) Suppose that $a b$ is right cancellative. Since:

$$
a b^{*} a b=b^{*}\left(a b^{*} a\right)=a^{*}\left(b^{*} a b\right)=a^{*}\left(b^{*} b a\right)=b a^{*} a b
$$

Since $a b$ is right cancellative, we get that $a b=b a$.

(5) Assume that $a b$ is cancellative. If $b^{*} x=b^{*} y, x, y \in S$, then:

$$
x^{*} a b=b^{*}\left(x^{*} a\right)=a^{*}\left(b^{*} x\right)=a^{*}\left(b^{*} y\right)=y^{*} a b
$$

Since $a b$ is cancellative, so $x=y$. This means that $b$ is left cancellative. Applying (1), we get that $b$ is cancellative.

(6) Assume that $a b$ is cancellative. Using (5), we know that $b$ is cancellative. Moreover, since $a b$ is cancellative, so $a b$ is right cancellative, applying (4) we get that $b a=a b$. Thus, $b a$ is cancellative, using (5) again, $a$ is cancellative.

(7) Suppose that $a$ and $a b$ are right cancellative, and $b$ is left cancellative. If $a^{*} x=a^{*} y, x, y \in S$, then (applying Proposition 1 (1)):

$$
b^{*}\left(x a^{*} a b\right)=b^{*}\left(b x^{*} a a\right)=b^{*}\left(a b^{*} a x\right)=b^{*}\left(a b^{*} a y\right)=b^{*}\left(y a^{*} a b\right) .
$$


Since $b$ is left cancellative, so $x a^{*} a b=y a^{*} a b$. Using the condition that $a b$ is right cancellative, it follows that $x a=y a$. Since $a$ is right cancellative, thus, $x=y$. Hence, $a$ is left cancellative. Therefore, $a$ is cancellative

(8) Suppose that $a$ and $a b$ are right cancellative, and $b$ is left cancellative. If $a b^{*} x=a b^{*} y, x, y \in S$, then:

$$
\begin{gathered}
b^{*}\left(x a^{*} a b\right)=a b^{*}\left(b^{*} x a\right)=a b^{*}\left(a^{*} b x\right)=a b^{*}\left(x^{*} a b\right)=a b^{*}\left(a b^{*} x\right)=a b^{*}\left(a b^{*} y\right)= \\
a b^{*}\left(y^{*} a b\right)=a b^{*}\left(a^{*} b y\right)=a b^{*}\left(b^{*} y a\right)=b^{*}\left(y a^{*} a b\right) .
\end{gathered}
$$

Since $b$ is left cancellative, so $x a^{*} a b=y a^{*} a b$. Using the condition that $a b$ is right cancellative, it follows that $x a=y a$. Since $a$ is right cancellative, thus, $x=y$. This means that $a b$ is left cancellative. From this and $a b$ is right cancellative, we know that $a b$ is cancellative.

Applying Theorem 1 we can get the following corollaries.

Corollary 1. Let $S$ be a $C A$-groupoid. Then the following asserts are equivalent:

(1) S is a left cancellative CA-groupoid;

(2) $S$ is a right cancellative $C A$-groupoid;

(3) $S$ is a cancellative and commutative semigroup;

(4) $S$ is a cancellative CA-groupoid.

Proof. (1) $\Rightarrow$ (2): It follows Theorem 1 (1).

$(2) \Rightarrow(3)$ : For any $a, b \in S$, then $a b \in S$. Since $S$ is right cancellative, then $a b$ is right cancellative. Applying Theorem $1(4), a b=b a$. This means that $S$ is commutative. By Proposition 2, we know that $S$ is a commutative semigroup. Moreover, since $S$ is right cancellative, so $S$ is left cancellative. Thus, $S$ is a cancellative and commutative semigroup.

(3) $\Rightarrow(4)$ : Obviously.

(4) $\Rightarrow$ (1): It follows from Definition 5 .

Corollary 2. Let $S$ be a $C A$-groupoid. If there exists a cancellative element in $S$, then the set $H=\{a \in S: a$ is cancellative\} is a sub CA-groupoid of $S$.

Proof. By the condition that there exists a cancellative element in $S$, we know that $H$ is not empty.

For any $a, b \in H$, then $a$ and $b$ are left and right cancellative. Applying Theorem 1 (2), we know that $a b$ is right cancellative. By Theorem 1 (8), $a b$ is cancellative. Thus $a b \in H$. It follows that $H$ is a sub CA-groupoid of $S$.

Corollary 3. Let $S$ be a $C A$-groupoid. If there exists a non-cancellative element in $S$, then the set $K=\{a \in S$ : $a$ is non-cancellative $\}$ is a sub $C A$-groupoid of $S$.

Proof. Obviously, $K$ is non-empty. For any $a, b \in K$, then $a$ and $b$ are non-cancellative. By Theorem 1 (5), we know that $a b$ is non-cancellative. Thus $a b \in K$. It follows that $K$ is a sub CA-groupoid of $S$.

The following example shows that a weak cancellative element maybe not a left (or right) cancellative element.

Example 3. Let $S=\{1,2,3,4,5\}$, and the operation * on $S$ is defined as Table 3, then $S$ is a CA-groupoid. It is easy to verify that 3 is weak cancellative, but 3 is not left (right) cancellative. 
Table 3. The operation * on $S$.

\begin{tabular}{llllll}
\hline$*$ & $\mathbf{1}$ & $\mathbf{2}$ & $\mathbf{3}$ & $\mathbf{4}$ & $\mathbf{5}$ \\
\hline $\mathbf{1}$ & 1 & 1 & 1 & 1 & 1 \\
$\mathbf{2}$ & 1 & 1 & 2 & 1 & 2 \\
$\mathbf{3}$ & 1 & 1 & 4 & 2 & 2 \\
$\mathbf{4}$ & 1 & 1 & 2 & 1 & 2 \\
$\mathbf{5}$ & 1 & 1 & 1 & 1 & 1 \\
\hline
\end{tabular}

Open Problem 1 (to prove or give a counterexample): Is any weak cancellative CA-groupoid necessarily cancellative?

Theorem 2. Let $S$ be a $C A$-groupoid and $a, b, c \in S$. Define on $S$ the relation $\sim$ as:

$$
a \sim b \Leftrightarrow a \text { and } b \text { are both cancellative or non - cancellative. }
$$

Then $\sim$ is an equivalence relation.

Proof. Suppose that $a$ is a cancellative element (or non-cancellative element) of CA-groupoid $S$. Then $a \sim a$. This means that $\sim$ is reflexive.

Suppose $a \sim b$. If $a$ and $b$ are cancellative, then $b \sim a$; if $a$ and $b$ are non-cancellative, then $b \sim a$. Thus $\sim$ is symmetric.

Next, suppose that $a \sim b$ and $b \sim c$. If $a$ and $b$ are cancellative, from $b \sim c$ we know that $c$ is cancellative, thus $a$ and $c$ are cancellative, i.e., $a \sim c$; if $a$ and $b$ are non-cancellative, from $b \sim c$ we know that $c$ is non-cancellative, thus $a$ and $c$ are non-cancellative, i.e., $a \sim c$. Thus $\sim$ is transitive.

Therefore, $\sim$ is an equivalence relation.

Example 4. Let $S=\{1,2,3,4\}$ and the operation * on $S$ is defined as Table 4, then $S$ is a CA-groupoid. Obviously, 1 and 2 are cancellative, 3 and 4 are non-cancellative. $H=\{1,2\}$ is a sub CA-groupoid of $S$.

Table 4. The operation * on $S$.

\begin{tabular}{lllll}
\hline$*$ & $\mathbf{1}$ & $\mathbf{2}$ & $\mathbf{3}$ & $\mathbf{4}$ \\
\hline $\mathbf{1}$ & 1 & 2 & 4 & 3 \\
$\mathbf{2}$ & 2 & 1 & 3 & 4 \\
$\mathbf{3}$ & 3 & 4 & 4 & 3 \\
$\mathbf{4}$ & 4 & 3 & 3 & 4 \\
\hline
\end{tabular}

Theorem 3. Let $\mathrm{S}_{1}, \mathrm{~S}_{2}$ are $C A$-groupoids, then the direct product $\mathrm{S}_{1} \times \mathrm{S}_{2}$ of $\mathrm{S}_{1}$ and $\mathrm{S}_{2}$ is a CA-groupoid. If $a \in S_{1}$, $b \in S_{2}, a$ and $b$ are cancellative, then $(a, b) \in S_{1} \times S_{2}$ is cancellative.

Proof. Suppose that $S_{1}$ and $S_{2}$ are CA-groupoids. By Proposition $5, S_{1} \times S_{2}$ is a CA-groupoid. Let $a \in S_{1}$, $b \in S_{2}, a$ and $b$ be cancellative. For any $\left(x_{1}, x_{2}\right),\left(y_{1}, y_{2}\right) \in S_{1} \times S_{2}$, if $(a, b) *\left(x_{1}, x_{2}\right)=(a, b) *\left(y_{1}, y_{2}\right)$, then:

$$
\begin{gathered}
\left(a x_{1}, b x_{2}\right)=\left(a y_{1}, b y_{2}\right) \\
a x_{1}=a y_{1}, b x_{2}=b y_{2} \\
x_{1}=y_{1}, x_{2}=y_{2} .(\text { since } a \text { and } b \text { are cancellative }) \\
\left(x_{1}, x_{2}\right)=\left(y_{1}, y_{2}\right) .
\end{gathered}
$$

hence, $(a, b)$ is cancellative. 


\section{Separability and Quasi-Cancellability of CA-Groupoids}

Definition 7. Let $S$ be a CA-groupoid. (1) $S$ is called to be left (right) separative, for all $x, y \in S$, if $x^{2}=x y$ and $y^{2}=y x\left(x^{2}=y x\right.$ and $\left.y^{2}=x y\right)$ imply $x=y$. (2) $S$ is called to be separative, if it is both left and right separative. (3) $S$ is called to be quasi-separative, if for all $x, y \in S, x^{2}=x y=y^{2}$ implies $x=y$.

Example 5. Let $S=\{1,2,3,4\}$. The operation $*$ on $S$ is defined as Table 5. Then $\left(S,{ }^{*}\right)$ is a separative CA-groupoid.

Table 5. The operation * on $S$.

\begin{tabular}{lllll}
\hline$*$ & $\mathbf{1}$ & $\mathbf{2}$ & $\mathbf{3}$ & $\mathbf{4}$ \\
\hline $\mathbf{1}$ & 4 & 2 & 1 & 1 \\
$\mathbf{2}$ & 2 & 2 & 2 & 2 \\
$\mathbf{3}$ & 1 & 2 & 3 & 4 \\
$\mathbf{4}$ & 1 & 2 & 4 & 4 \\
\hline
\end{tabular}

Example 6. Let $S=\{1,2,3,4\}$. The operation ${ }^{*}$ on $S$ is defined as Table 6 . Then $\left(S,{ }^{*}\right)$ is a quasi-separative CA-groupoid.

Table 6. The operation * on $S$.

\begin{tabular}{lllll}
\hline$*$ & $\mathbf{1}$ & $\mathbf{2}$ & $\mathbf{3}$ & $\mathbf{4}$ \\
\hline $\mathbf{1}$ & 4 & 3 & 2 & 1 \\
$\mathbf{2}$ & 3 & 2 & 3 & 2 \\
$\mathbf{3}$ & 2 & 3 & 2 & 3 \\
$\mathbf{4}$ & 1 & 2 & 3 & 4 \\
\hline
\end{tabular}

Theorem 4. Let S be a CA-groupoid. Then the following asserts are equivalent:

(1) $S$ is separative;

(2) $S$ is left separative;

(3) $S$ is right separative;

(4) $S$ is quasi-separative.

Proof. Obviously, (1) $\Rightarrow(2)$, by Definition 7.

$(2) \Rightarrow(3)$ : Suppose that $S$ is left separative. For any $x, y \in S$, if $x^{2}=y x$ and $y^{2}=x y$, then (by Proposition $1(1))$ :

$$
\begin{gathered}
(x y)^{2}=(x y)(x y)=(x y) y^{2}=(x y)(y y)=(y x)(y y)=x^{2}(y y)=(x x)(y y)=(y x)(y x)=(x y)(y x) \\
(y x)^{2}=(y x)(y x)=(x y)(y x)=(x x)(y y)=x^{2} y^{2}=(y x)(x y) .
\end{gathered}
$$

Since $S$ is left separative, by Definition 7 we have $x y=y x$. From this, using $x^{2}=y x$ and $y^{2}=x y$, we get that $x^{2}=x y$ and $y^{2}=y x$. Applying the condition that $S$ is left separative, by Definition 7 again, we have $x=y$. This means that $S$ is right separative.

$(3) \Rightarrow(4)$ : Suppose that $S$ is right separative. For any $x, y \in S$, if $x^{2}=x y=y^{2}$, then (by Proposition $1(1))$ :

$$
\begin{gathered}
(x y)^{2}=(x y)(x y)=x^{2}(x y)=(x x)(x y)=(y x)(x x)=(y x) x^{2}=(y x)(x y) \\
(y x)^{2}=(y x)(y x)=(x y)(y x) .
\end{gathered}
$$


Since $S$ is right separative, by Definition 7 we have $x y=y x$. From this, using $x^{2}=x y=y^{2}$, we get that $x^{2}=y x$ and $y^{2}=x y$. Applying the condition that $S$ is right separative, by Definition 7 again, we have $x=y$. This means that $S$ is quasi-separative.

$(4) \Rightarrow(1)$ Suppose that $S$ is quasi-separative. For any $x, y \in S$, then (by Proposition 1 (1)):

$$
\begin{aligned}
& (x y)^{2}=(x y)(x y)=(y x)(x y)=(y y)(x x)=y^{2} x^{2} ; \\
& (y x)^{2}=(y x)(y x)=(x y)(y x)=(x x)(y y)=x^{2} y^{2} .
\end{aligned}
$$

Moreover,

$$
\begin{gathered}
{[(y x)(x y)]^{2}=[(y x)(x y)][(y x)(x y)]=[(y x)(x y)][(y y)(x x)]=[(y x)(x y)]\left(y^{2} x^{2}\right)=[(y x)(x y)](x y)^{2}=} \\
{[(y x)(x y)][(x y)(x y)]=[(x y)(y x)][(x y)(x y)]=[(x x)(y y)][(x y)(x y)]=\left(x^{2} y^{2}\right)[(x y)(x y)]=} \\
(y x)^{2}[(x y)(x y)]=[(y x)(y x)][(x y)(x y)]=[(x y)(y x)][(x y)(y x)]=[(x y)(y x)]^{2} . \\
\text { If } x^{2}=x y \text { and } y^{2}=y x \text {, then } \\
\left(y^{4}\right)^{2}=\left(y^{2} y^{2}\right)^{2}=[(y x)(y x)]^{2}=[(x y)(y x)]^{2}=[(y x)(x y)]^{2}=\left(y^{2} x^{2}\right)^{2}=\left(y^{2} x^{2}\right)\left(y^{2} x^{2}\right)=\left(x^{2} y^{2}\right)\left(y^{2} x^{2}\right)=\left(x^{2} x^{2}\right)\left(y^{2} y^{2}\right)=x^{4} y^{4} ; \\
\left(x^{4}\right)^{2}=\left(x^{2} x^{2}\right)^{2}=[(x y)(x y)]^{2}=[(y x)(x y)]^{2}=[(y y)(x x)]^{2}=\left(y^{2} x^{2}\right)^{2}=\left(y^{2} x^{2}\right)\left(y^{2} x^{2}\right)=\left(x^{2} y^{2}\right)\left(y^{2} x^{2}\right)=\left(x^{2} x^{2}\right)\left(y^{2} y^{2}\right)=x^{4} y^{4} .
\end{gathered}
$$

From this, applying the condition that $S$ is quasi-separative, we get that $x^{4}=y^{4}$. Thus,

$$
(x y)^{2}=\left(x^{2}\right)^{2}=x^{4}=y^{4}=\left(y^{2}\right)^{2}=(y x)^{2}=(y x)(y x)=(x y)(y x) .
$$

That is, $(x y)^{2}=(x y)(y x)=(y x)^{2}$. Since $S$ is quasi-separative, by Definition 7 we have $x y=y x$. From this, using $x^{2}=x y$ and $y^{2}=y x$, we have $x^{2}=x y=y^{2}$. Applying the condition that $S$ is quasi-separative, by Definition 7 again, we have $x=y$. This means that $\mathrm{S}$ is left separative.

Similarly, we can prove that $S$ is right separative. Therefore, $S$ is separative by Definition 7 .

Proposition 6. Let $S$ be a CA-groupoid. If $S$ is cancellative, then $S$ is separative.

Proof. Assume that $S$ is cancellative. For any $x, y \in S$, if $x^{2}=x y=y^{2}$, then $x x=x y$ and $x y=y y$. Using cancellability of $S$, we have $x=y$. This means that $S$ is separative.

Similarly, we can prove that $S$ is separative when $S$ is left (or right) cancellative.

The following example shows that a separative CA-groupoid maybe not a left (or right) cancellative CA-groupoid.

Example 7. Let $S=\{1,2,3,4\}$. The operation * on $S$ is defined as Table 7 . Then $\left(S,{ }^{*}\right)$ is a separative CA-groupoid, but $S$ isn't cancellative, since $1^{*} 1=2^{*} 1,1 \neq 2$.

Table 7. The operation * on $S$.

\begin{tabular}{lllll}
\hline$*$ & $\mathbf{1}$ & $\mathbf{2}$ & $\mathbf{3}$ & $\mathbf{4}$ \\
\hline $\mathbf{1}$ & 1 & 2 & 3 & 1 \\
$\mathbf{2}$ & 1 & 4 & 3 & 2 \\
$\mathbf{3}$ & 3 & 3 & 3 & 3 \\
$\mathbf{4}$ & 1 & 2 & 3 & 4 \\
\hline
\end{tabular}

Definition 8. Let $S$ be a $C A$-groupoid. $S$ is called a $C A$-band, if for all $a \in S, a a=a$; $S$ is called $C A$-3-band, if for all $a \in S, a^{*} a a=a a^{*} a=a$. 
Definition 9. Let $S$ be a $C A$-groupoid. $S$ is called to be left(right) quasi-cancellative, for all $x, y \in S$, if $x=x y$ and $y^{2}=y x\left(x=y x\right.$ and $\left.y^{2}=x y\right)$ imply $x=y$. $S$ is called quasi-cancellative, if it is both left and right quasicancellative.

Example 8. Let $S=\{1,2,3,4\}$. The operation * on $S$ is defined as Table 8. Then $(S, *)$ is a quasi-cancellative CA-groupoid.

Table 8. The operation * on $S$.

\begin{tabular}{lllll}
\hline$*$ & $\mathbf{1}$ & $\mathbf{2}$ & $\mathbf{3}$ & $\mathbf{4}$ \\
\hline $\mathbf{1}$ & 1 & 1 & 1 & 1 \\
$\mathbf{2}$ & 1 & 2 & 1 & 1 \\
$\mathbf{3}$ & 1 & 1 & 3 & 4 \\
$\mathbf{4}$ & 1 & 1 & 4 & 3 \\
\hline
\end{tabular}

Theorem 5. Let $S$ be a CA-groupoid. If $S$ is left quasi-cancellative, then $S$ is right quasi-cancellative.

Proof. Suppose that $S$ is left quasi-cancellative. For any $x, y \in S$, if $x=y x$ and $y^{2}=x y$, then (by Proposition $1(1))$ :

$$
\begin{gathered}
x^{2}=(y x)(y x)=(x y)(y x)=(x x)(y y)=x^{2} y^{2} ; \\
\left(y^{2}\right)^{2}=y^{2} y^{2}=(x y)(x y)=(y x)(x y)=(y y)(x x)=y^{2} x^{2} .
\end{gathered}
$$

From this, applying the condition that $S$ is left quasi-cancellative, we get that $x^{2}=y^{2}$. Thus:

$$
\begin{gathered}
x y=y^{2}=x^{2}=(y x)(y x)=(x y)(y x) ; \\
(y x)^{2}=(y x)(y x)=x(y x)=x(x y)=(y x)(x y) .
\end{gathered}
$$

From this, applying the condition that $S$ is left quasi-cancellative and Definition 9 again, we get that $x y=y x$. Hence, using the condition that $x=y x$ and $y^{2}=x y$, we have $x=x y$ and $y^{2}=y x$, applying the definition of left quasi-cancellative, we get that $x=y$. Therefore, $S$ is right quasi-cancellative.

Open Problem 2 (to prove or give a counterexample): Is any right quasi-cancellative CA-groupoid necessarily left quasi-cancellative?

Theorem 6. The following asserts are true:

(1) Every CA-band is quasi-cancellative.

(2) Every CA-3-band is quasi-cancellative.

(3) Every quasi-separative CA-groupoid is quasi-cancellative;

(4) Every separative (or left-, right-separative) CA-groupoid is quasi-cancellative.

Proof. (1) Let $S$ be a CA-band. For any $x, y \in S$, if $x=x y$ and $y^{2}=y x$, then (by Definition 8) $x=x^{2}$, $y=y^{2}$. It follows that:

$$
x=x^{2}=(x y)(x y)=(y x)(x y)=y^{2}(x y)=y(x y)=y x=y^{2}=y .
$$

This means that $S$ is left quasi-cancellative. Applying Theorem 5, we know that $S$ is right quasicancellative. Hence, $S$ is quasi-cancellative. 
(2) Let $S$ be a CA-3-band. For any $x, y \in S$, if $x=x y$ and $y^{2}=y x$, then (by Definition 8) $x=x x^{2}=$ $x^{2} x, y=y y^{2}=y^{2} y$. Furthermore:

$$
\begin{gathered}
y^{2}=y x=y(x y)=y(y x)=y y^{2}=y, \\
x=x y=x\left(y y^{2}\right)=y^{2}(x y)=y^{2} x=y x=y^{2}=y .
\end{gathered}
$$

Thus, $S$ is left quasi-cancellative. Applying Theorem 5 , we get that $S$ is right quasi-cancellative. Hence, $S$ is quasi-cancellative.

(3) Let $S$ be a quasi-separative CA-groupoid. For any $x, y \in S$, if $x=x y$ and $y^{2}=y x$, then:

$$
x^{2}=x x=x(x y)=y(x x)=x(y x)=x y^{2}=x(y y)=y(x y)=y x=y^{2}
$$

That is, $y^{2}=y x=x^{2}$. By Definition 7 we have $x=y$. This means that $S$ is left quasi-cancellative. Applying Theorem 5, we get that $S$ is right quasi-cancellative. Hence, $S$ is quasi-cancellative.

(4) It follows from (3) and Theorem 4.

Example 9. Let $S=\{1,2,3,4,5\}$. The operation * on $S$ is defined as Table 9. Then $\left(S,{ }^{*}\right)$ is a quasi-cancellative CA-groupoid, $S$ isn't separative, because $2^{*} 2=2^{*} 4=3,4^{*} 4=4^{*} 2=3$, but $2 \neq 4$.

Table 9. The operation * on $S$.

\begin{tabular}{llllll}
\hline$*$ & $\mathbf{1}$ & $\mathbf{2}$ & $\mathbf{3}$ & $\mathbf{4}$ & $\mathbf{5}$ \\
\hline $\mathbf{1}$ & 3 & 3 & 5 & 3 & 3 \\
$\mathbf{2}$ & 3 & 3 & 5 & 3 & 3 \\
$\mathbf{3}$ & 4 & 4 & 3 & 5 & 5 \\
$\mathbf{4}$ & 3 & 3 & 5 & 3 & 3 \\
$\mathbf{5}$ & 3 & 3 & 5 & 3 & 3 \\
\hline
\end{tabular}

Definition 10. Let $\left(S,{ }^{*}\right)$ be a $C A$-groupoid. $S$ is called to be power-cancellative, if for all $x, y \in S, x^{2}=y^{2}$ implies $x=y$.

Example 10. Let $S=\{1,2,3,4,5\}$. The operation ${ }^{*}$ on $S$ is defined as Table 10. Then $\left(S,{ }^{*}\right)$ is a power-cancellative CA-groupoid, $S$ isn't cancellative, because $1^{*} 2=1 * 3$, but $2 \neq 3$.

Table 10. The operation * on $S$.

\begin{tabular}{llllll}
\hline$*$ & $\mathbf{1}$ & $\mathbf{2}$ & $\mathbf{3}$ & $\mathbf{4}$ & $\mathbf{5}$ \\
\hline $\mathbf{1}$ & 1 & 1 & 1 & 4 & 5 \\
$\mathbf{2}$ & 1 & 2 & 1 & 4 & 5 \\
$\mathbf{3}$ & 1 & 1 & 3 & 4 & 5 \\
$\mathbf{4}$ & 4 & 4 & 4 & 5 & 1 \\
$\mathbf{5}$ & 5 & 5 & 5 & 1 & 4 \\
\hline
\end{tabular}

Example 11. Let $S=\{1,2,3,4\}$. The operation ${ }^{*}$ on $S$ is defined as Table 11. Then $\left(S,{ }^{*}\right)$ is a cancellative CA-groupoid, $S$ isn't power-cancellative, because $1^{2}=2^{2}=1$, but $1 \neq 2$. 
Table 11. The operation * on $S$.

\begin{tabular}{lllll}
\hline$*$ & $\mathbf{1}$ & $\mathbf{2}$ & $\mathbf{3}$ & $\mathbf{4}$ \\
\hline $\mathbf{1}$ & 1 & 2 & 3 & 4 \\
$\mathbf{2}$ & 2 & 1 & 4 & 3 \\
$\mathbf{3}$ & 3 & 4 & 2 & 1 \\
$\mathbf{4}$ & 4 & 3 & 1 & 2 \\
\hline
\end{tabular}

Theorem 7. Let $S$ be a CA-groupoid. If $S$ is power-cancellative, then:

(1) $S$ is commutative, and $S$ is a commutative semigroup.

(2) $S$ is separative.

Proof. (1) Suppose that $S$ is power-cancellative. For any $x, y \in S$, since (by Proposition 1 (1)):

$$
\begin{aligned}
& (x y)^{2}=(x y)(x y)=(y x)(x y)=(y y)(x x)=y^{2} x^{2} \\
& (y x)^{2}=(y x)(y x)=(x y)(y x)=(x x)(y y)=x^{2} y^{2} .
\end{aligned}
$$

Moreover,

$$
\begin{gathered}
{[(y x)(x y)]^{2}=[(y x)(x y)][(y x)(x y)]=[(y x)(x y)][(y y)(x x)]=[(y x)(x y)]\left(y^{2} x^{2}\right)=[(y x)(x y)](x y)^{2}=} \\
{[(y x)(x y)][(x y)(x y)]=[(x y)(y x)][(x y)(x y)]=[(x x)(y y)][(x y)(x y)]=\left(x^{2} y^{2}\right)[(x y)(x y)]=} \\
(y x)^{2}[(x y)(x y)]=[(y x)(y x)][(x y)(x y)]=[(x y)(y x)][(x y)(y x)]=[(x y)(y x)]^{2}
\end{gathered}
$$

Applying the condition that $S$ is power-cancellative, we get that $(y x)(x y)=(x y)(y x)$. Thus:

$$
(x y)^{2}=y^{2} x^{2}=(y x)(x y)=(x y)(y x)=x^{2} y^{2}=(y x)^{2}
$$

By Definition 10, we have $x y=y x$. This means that $S$ is commutative, and $S$ is a commutative semigroup (by Proposition 2).

(2) Assume that $S$ is power-cancellative. For any $x, y \in S$, if $x^{2}=x y=y^{2}$, then (by Definition 10), $x=y$. This means that $S$ is quasi-separative. Applying Theorem 4, we know that $S$ is separative.

\section{Variant CA-Groupoids}

In this section, we focus on a special class of CA-groupoids, which are called variant CA-groupoids. The reasons why we want to discuss this kind of CA-groupoids are that: (1) it is closely related to the generalized unit element (i.e., quasi right unit element), and it is the closest to the commutative semigroup (see Example 12 and Example 13 below); (2) this kind of CA-groupoids has many interesting properties, and it can constructed from any commutative semigroup, please refer to the following Theorem 9; (3) the research this kind of CA-groupoids is of great significance to study some special rings and semirings. See literature [7-9] and Example 14 and Example 15 below.

Definition 11. Let $\left(S,{ }^{*}\right)$ be a $C A$-groupoid. $S$ is called a variant $C A$-groupoid, if exist e $\in$, such that for all $x \in S-\{e\}, x e=x$ and $e^{2} \neq e$. Where, $e$ is called a quasi-right unite element of $S$.

Example 12. Let $S=\{1,2,3,4,5\}$, The operation * on $S$ is defined as Table 12, then $\left(S,{ }^{*}\right)$ is a variant $C A$-groupoid and 1 is a quasi-right unit element in S. Obviously, $S$ isn't commutative. 
Table 12. The operation * on $S$.

\begin{tabular}{llllll}
\hline$*$ & $\mathbf{1}$ & $\mathbf{2}$ & $\mathbf{3}$ & $\mathbf{4}$ & $\mathbf{5}$ \\
\hline $\mathbf{1}$ & 3 & 3 & 3 & 4 & 4 \\
$\mathbf{2}$ & 2 & 3 & 3 & 4 & 4 \\
$\mathbf{3}$ & 3 & 3 & 3 & 4 & 4 \\
$\mathbf{4}$ & 4 & 4 & 4 & 4 & 4 \\
$\mathbf{5}$ & 5 & 4 & 4 & 4 & 4 \\
\hline
\end{tabular}

Looking at the above example carefully, we find that: (1) the element 1 as a quasi-right unit element of $S$, does not appear in the operation table; (2) in the operation table, the first row is the same as the third row; (3) if we change the first row of the operation table to $\{1,2,3,4,5\}$, we will get a commutative semigroup $(S,+)$ (as shown in Table 13). These are all interesting phenomena. Later, we will analyze the characteristics of variant CA-groupoids.

Table 13. A Commutative semigroup $(S,+)$ corresponding to $(S, *)$.

\begin{tabular}{llllll}
\hline+ & $\mathbf{1}$ & $\mathbf{2}$ & $\mathbf{3}$ & $\mathbf{4}$ & $\mathbf{5}$ \\
\hline $\mathbf{1}$ & 1 & 2 & 3 & 4 & 5 \\
$\mathbf{2}$ & 2 & 3 & 3 & 4 & 4 \\
$\mathbf{3}$ & 3 & 3 & 3 & 4 & 4 \\
$\mathbf{4}$ & 4 & 4 & 4 & 4 & 4 \\
$\mathbf{5}$ & 5 & 4 & 4 & 4 & 4 \\
\hline
\end{tabular}

Example 13. Let $S=\{1,2,3,4,5\}$, The operation * on $S$ is defined as Table 14 , then $\left(S,{ }^{*}\right)$ is a variant $C A$-groupoid and 5 is a quasi-right unit element in S. Obviously, $S$ is commutative.

Table 14. The operation * on $S$.

\begin{tabular}{llllll}
\hline$*$ & $\mathbf{1}$ & $\mathbf{2}$ & $\mathbf{3}$ & $\mathbf{4}$ & $\mathbf{5}$ \\
\hline $\mathbf{1}$ & 1 & 1 & 1 & 1 & 1 \\
$\mathbf{2}$ & 1 & 4 & 2 & 3 & 2 \\
$\mathbf{3}$ & 1 & 2 & 3 & 4 & 3 \\
$\mathbf{4}$ & 1 & 3 & 4 & 2 & 4 \\
$\mathbf{5}$ & 1 & 2 & 3 & 4 & 3 \\
\hline
\end{tabular}

If we change the last row of the operation table to $\{1,2,3,4,5\}$, we will get a commutative semigroup $(S,+)$ (as shown in Table 15).

Table 15. A Commutative semigroup $(S,+)$ corresponding to $(S, *)$.

\begin{tabular}{llllll}
\hline+ & $\mathbf{1}$ & $\mathbf{2}$ & $\mathbf{3}$ & $\mathbf{4}$ & $\mathbf{5}$ \\
\hline $\mathbf{1}$ & 1 & 1 & 1 & 1 & 1 \\
$\mathbf{2}$ & 1 & 4 & 2 & 3 & 2 \\
$\mathbf{3}$ & 1 & 2 & 3 & 4 & 3 \\
$\mathbf{4}$ & 1 & 3 & 4 & 2 & 4 \\
$\mathbf{5}$ & 1 & 2 & 3 & 4 & 5 \\
\hline
\end{tabular}


Example 14. Let:

$$
S=\left\{\left(\begin{array}{ll}
a & 0 \\
0 & 0
\end{array}\right): \text { a is a integral number }\right\} \cup\left\{\left(\begin{array}{cc}
1 & 0 \\
0 & 1
\end{array}\right),\left(\begin{array}{cc}
1 & 0 \\
0 & -1
\end{array}\right)\right\}
$$

Define the operation * on $S$ is the common matrix multiplication, then $\left(S,{ }^{*}\right)$ is a variant $C A$-groupoid and $\left(\begin{array}{cc}1 & 0 \\ 0 & -1\end{array}\right)$ is a quasi-right unit element in S. Moreover, we define the addition operation + on $S$ as following: for any $x, y \in S$, denote $S_{1}=\left\{\left(\begin{array}{ll}a & 0 \\ 0 & 0\end{array}\right):\right.$ a is a integral number $\}, S_{2}=\left\{\left(\begin{array}{ll}1 & 0 \\ 0 & 1\end{array}\right),\left(\begin{array}{cc}1 & 0 \\ 0 & -1\end{array}\right)\right\}$,

(1) if $x, y \in S_{1}, x+y$ is common matrix addition;

(2) if $x \in S_{1}$ and $y \in S_{2}, x+y=\left(\begin{array}{cc}a+1 & 0 \\ 0 & 0\end{array}\right)$, where $y=\left(\begin{array}{ll}a & 0 \\ 0 & 0\end{array}\right)$;

(3) if $x \in S_{2}$ and $y \in S_{1}, x+y=y+x(\sec (2))$;

(4) if $x=y \in S_{2}, x+y=\left(\begin{array}{ll}0 & 0 \\ 0 & 0\end{array}\right)$;

(5) if $x, y \in S_{2}$ and $x \neq y, x+y=\left(\begin{array}{ll}2 & 0 \\ 0 & 0\end{array}\right)$.

Then $(S,+)$ is a commutative group, and $\left(S ;+{ }^{*}\right)$ is a ring, that is, $(x+y)^{*} z=x^{*} z+y^{*} z$ and $z^{*}(x+y)=$ $z^{*} x+z^{*} y$, for any $x, y, z \in S$.

Example 15. Let $S=\{1,2,3,4,5,6\}$, The operation * on $S$ is defined as Table 16, then $\left(S,{ }^{*}\right)$ is a variant CA-groupoid and 1 is a quasi-right unit element in $S$. Obviously, $S$ is not commutative.

Table 16. The operation * on $S$.

\begin{tabular}{lllllll}
\hline$*$ & $\mathbf{1}$ & $\mathbf{2}$ & $\mathbf{3}$ & $\mathbf{4}$ & $\mathbf{5}$ & $\mathbf{6}$ \\
\hline $\mathbf{1}$ & 3 & 3 & 3 & 4 & 4 & 6 \\
$\mathbf{2}$ & 2 & 3 & 3 & 4 & 4 & 6 \\
$\mathbf{3}$ & 3 & 3 & 3 & 4 & 4 & 6 \\
$\mathbf{4}$ & 4 & 4 & 4 & 4 & 4 & 6 \\
$\mathbf{5}$ & 5 & 4 & 4 & 4 & 4 & 6 \\
$\mathbf{6}$ & 6 & 6 & 6 & 6 & 6 & 6 \\
\hline
\end{tabular}

Moreover, we define the addition operation + on $S$ as Table 17 or Table 18 , then $(S,+)$ is a commutative semigroup with unite 6 . We can verify that $(x+y)^{*} z=x^{*} z+y^{*} z$ for any $x, y, z$ in $S$, so $\left(S ;+,{ }^{*}\right)$ is a semiring (for the theory of semirings, please see the monograph [33-35]).

Table 17. A Commutative monoid $(S,+)$.

\begin{tabular}{lllllll}
\hline+ & $\mathbf{1}$ & $\mathbf{2}$ & $\mathbf{3}$ & $\mathbf{4}$ & $\mathbf{5}$ & $\mathbf{6}$ \\
\hline $\mathbf{1}$ & 1 & 1 & 1 & 1 & 1 & 1 \\
$\mathbf{2}$ & 1 & 2 & 3 & 3 & 3 & 2 \\
$\mathbf{3}$ & 1 & 3 & 3 & 3 & 3 & 3 \\
$\mathbf{4}$ & 1 & 3 & 3 & 4 & 5 & 4 \\
$\mathbf{5}$ & 1 & 4 & 3 & 5 & 5 & 5 \\
$\mathbf{6}$ & 1 & 2 & 3 & 4 & 5 & 6 \\
\hline
\end{tabular}


Table 18. Another Commutative monoid $(S,+)$.

\begin{tabular}{lllllll}
\hline+ & $\mathbf{1}$ & $\mathbf{2}$ & $\mathbf{3}$ & $\mathbf{4}$ & $\mathbf{5}$ & $\mathbf{6}$ \\
\hline $\mathbf{1}$ & 1 & 2 & 1 & 4 & 5 & 1 \\
$\mathbf{2}$ & 2 & 2 & 2 & 4 & 5 & 2 \\
$\mathbf{3}$ & 1 & 2 & 3 & 4 & 5 & 3 \\
$\mathbf{4}$ & 4 & 4 & 4 & 4 & 5 & 4 \\
$\mathbf{5}$ & 5 & 5 & 5 & 5 & 4 & 5 \\
$\mathbf{6}$ & 1 & 2 & 3 & 4 & 5 & 6 \\
\hline
\end{tabular}

Theorem 8. Let $S$ be a variant $C A$-groupoid:

(1) If e is a quasi-right unit element of $S$ and ee $=a, a \in S$, then ex $=a x$ for all $x \in S$.

(2) The quasi-right unite element is unique in $S$.

Proof. (1) Let $e$ be a quasi-right unit element of $S$ and $e e=a, a \in S$. By Definition 11, we know that $a \neq e$. For any $x \in S$, if $x=e$, then $e x=e e=a=a e=a x$; if $x \neq e$, then (by Definition 11):

$$
\begin{aligned}
e x=e^{*} x e=e^{*} e x=x^{*} e e= & x a=x e^{*} a e=e^{*}\left(x e^{*} a\right)=a^{*}\left(e^{*} x e\right)=a^{*}\left(e^{*} e x\right)=a^{*}\left(x^{*} e e\right)=a^{*} x a=a^{*} a x \\
& =x^{*} a a=x^{*}\left(a^{*} e e\right)=x^{*}\left(e^{*} a e\right)=a e^{*} x e=a x .
\end{aligned}
$$

hence, $e x=a x$ for all $x \in S$.

(2) Suppose that $s$ and $t$ are quasi-right unit elements of $S, s \neq t$. From Definition 11 we know that $s s \neq s$ and $t t \neq t$. Since:

$$
s=s t=s t^{*} t s=s^{*}\left(s t^{*} t\right)=t^{*}\left(s^{*} s t\right)=t^{*}\left(t^{*} s s\right)=t^{*}\left(s^{*} t s\right)=t^{*} s t=t s=t .
$$

This means that the quasi-right unit element is unique in $S$.

Obviously, let $S=\{a\}$ and $(S, *)$ is a CA-groupoid, then $S$ isn't a variant CA-groupoid. Let $S=\{a, b\}$ and $\left(S,{ }^{*}\right)$ is a variant CA-groupoid, denote the quasi-right unit element $e=a($ or $b)$, then for any $x, y \in S$, we have $x y=b$ (or $a)$.

Through the study of the variant CA-groupoid, we give the following construction method, that is to say, on the basis of a commutative semigroup, a variant CA-groupoid is formed by adding an element which does not intersect with it, and a variant CA-groupoid can also be decomposed to obtain a commutative semigroup and an independent element.

Theorem 9. The following asserts are true:

(1) Let $S$ be a variant $C A$-groupoid and e is the quasi-right unite element on $S$, then $S_{1}=S-\{e\}$ is a commutative semigroup.

(2) Let $S$ be a commutative monoid with unit element $e$ and $a$ is an element such that $\{a\} \cap S=\varnothing$, then $S_{2}=$ $S \cup\{a\}$ is a variant $C A$-groupoid if define $x a=x, a x=e x, a a=e$, for all $x \in S$.

Proof. (1) Suppose that $S$ is a variant CA-groupoid and $e$ is the quasi-right unit element of $S$, if $\exists x, y \in$ $S_{1}=S-\{e\}$ such that $x y=e$, then for all $a \in S-\{e\}, a^{*} x y=a e=a$, so we have:

$$
e e=e^{*} x y=y^{*} e x=x^{*} y e=x y=e .
$$


This conclusion contradicts Definition 11. Hence, for all $x, y \in S-\{e\}, x y \neq e$, in other words, $S-\{e\}$ is closed, that is, $S-\{e\}$ is a sub CA-groupoid of $S$. Moreover, for all $x, y \in S_{1}=S-\{e\}$, applying Theorem 8 $(1), e x=(e e) x$, and:

$$
x y=x^{*} y e=e^{*} x y=y^{*} e x=y^{*}\left(e e^{*} x\right)=x^{*}\left(y^{*} e e\right)=x^{*}\left(e^{*} y e\right)=y e^{*} x e=y x
$$

hence $S-\{e\}$ is commutative, then $S-\{e\}$ is a commutative semigroup (by Proposition 2).

(2) On the other hand, suppose that $S$ is commutative monoid with unit element $e$. Let $a$ be an element such that $\{a\} \cap S=\varnothing$, denote $S_{2}=S \cup\{a\}$. Define a new binary operation $\bullet$ on $S_{2}$ :

for any $x, y \in S_{2}$, if $x, y \in \mathrm{S}$, then $x \bullet y=x^{*} y$; if $x \in S$, then $x \bullet a=x, a \bullet x=e \bullet x, a \bullet a=e$.

Obviously, $\left(S_{2}, \bullet\right)$ is a groupoid. For all $x, y, z \in S$, by the definition of operation $\bullet$ we have:

$$
\begin{gathered}
x \bullet y z=x^{*} y z=z^{*} x y=z \bullet x y, \\
a \bullet a a=a \bullet a a, \\
x \bullet a a=x e=e x=a x=a \bullet x a, \\
a \bullet x a=a x=b x=x e=x \bullet e e=e \bullet e x=a \bullet a x, \\
a \bullet a x=e \bullet e x=e \bullet x e=x e \bullet e x \bullet e e=x e=x \bullet a a, \\
y \bullet a x=a x \bullet y=e x \bullet y=x y=x \bullet y a, \\
x \bullet y a=x y=e \bullet x y=a \bullet x y, \\
a \bullet x y=(a \bullet x a) \bullet y a=y a \bullet(a \bullet x a)=y \bullet a x .
\end{gathered}
$$

thus, $\left(S_{2}, \bullet\right)$ is a variant CA-groupoid with the quasi-right unit element $a$.

Applying Definition 11 and Definition 9 we can easy to verify that the following proposition is true.

Proposition 7. (1) If $S$ is a variant $C A$-groupoid, then $S$ isn't cancellative. (2) If $S$ is a cancellative $C A$-groupoid, then $S$ isn't a variant $C A$-groupoid.

From Theorem 9, Proposition 7, Examples 12 15, we have Figure 1.

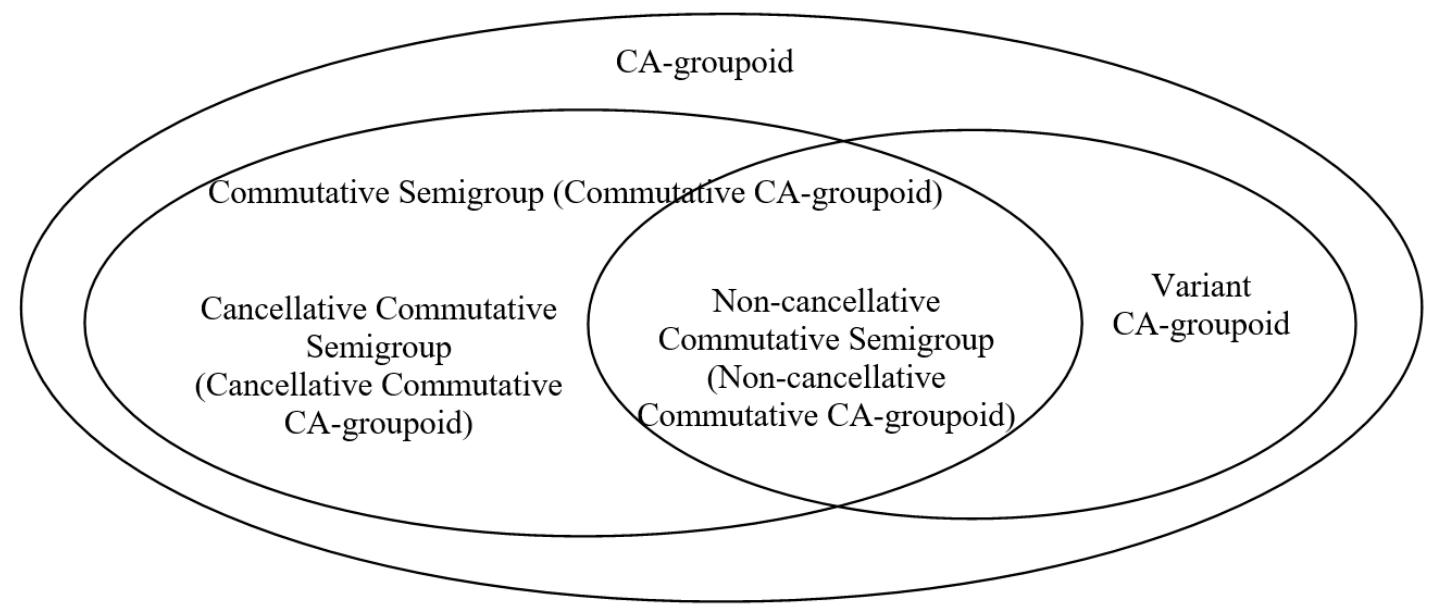

Figure 1. The relationships among some CA-groupoids. 
Theorem 10. Let $S$ be a variant $C A$-groupoid and e be a quasi-right unit element of $S$. Denote $a=e e, b=a a$. Then the following asserts are true:

(1) If $b=a$, then $\{e, a\}$ is a variant sub $C A$-groupoid of $S$;

(2) If $b \neq a$, then $\{e, a, b\}$ is a variant sub $C A$-groupoid of $S$.

Proof. (1) Suppose $b=a$. For the set $\{e, a\}$, since (by Theorem 8):

$$
e e=a, a a=b=a, a e=a, e a=e e^{*} a=a a=a .
$$

It follows that $\{e, a\}$ is closed on the operation *. Thus, $\{e, a\}$ is a variant sub CA-groupoid with quasi-right unit element $e$.

(2) Assume $b \neq a$. By Theorem 9 (1), for all $x, y \in S-\{e\}, x y=y x$. For the set $\{e, a, b\}$, since (by Theorem 8):

$$
\begin{gathered}
e e=a, a a=b, a e=a, e a=e e^{*} a=a a=b ; \\
e b=e^{*} a a=a^{*} e a=a^{*} a e=a a=b, b e=b ; \\
b a=a b=e e^{*} a a=a^{*}\left(e e^{*} a\right)=a^{*}\left(a^{*} e e\right)=a^{*}\left(e^{*} a e\right)=a e^{*} a e=a a=b .
\end{gathered}
$$

Thus, $\{e, a, b\}$ is closed about ${ }^{*}$, so $\{e, a, b\}$ is a variant sub CA-groupoid of $S$.

Theorem 11. Let $\left(S_{1},{ }_{1}\right)$ and $\left(S_{2},{ }^{*}\right)$ be two variant $C A$-groupoids, $e_{1}$ and $e_{2}$ are quasi-right unit elements of $\left(S_{1},{ }_{1}\right)$ and $\left(S_{2},{ }_{2}^{*}\right), S_{1} \cap S_{2}=\{e\}\left(e=e_{1}=e_{2}\right)$. Denote $S=S_{1} \cup S_{2}$, and define the operation ${ }^{*}$ on $S$ as follows:

(i) if $a, b \in S_{1}$, then $a^{*} b=a^{*}{ }_{1} b$;

(ii) if $a, b \in S_{2}$, then $a^{*} b=a^{*}{ }_{2} b$;

(iii) if $a \in S_{1}-\{e\}, b \in S_{2}-\{e\}$, then $a^{*} b=b$;

(iv) if $a \in S_{2}-\{e\}, b \in S_{1}-\{e\}$, then $a^{*} b=a$.

Then $(S, *)$ is a variant $C A$-groupoid with the quasi-right unite $e$.

Proof. It is only necessary to prove that the cyclic associative law hold in $\left(S,{ }^{*}\right)$, that is, $a^{*}\left(b^{*} c\right)=c^{*}\left(a^{*} b\right)$ for all $a, b, c \in S$. We will discuss the following situations separately:

(1) If $a, b, c \in S_{1}$, or $a, b, c \in S_{2}$, then $a^{*}\left(b^{*} c\right)=c^{*}\left(a^{*} b\right)$;

(2) If $a \in S_{1}-\{e\}, b \in S_{2}-\{e\}$ and $c \in S_{2}-\{e\}$, then $a^{*}\left(b^{*} c\right)=b^{*} c=c^{*} b=c^{*}\left(a^{*} b\right)$;

(3) If $a \in S_{2}-\{e\}, b \in S_{1}-\{e\}$ and $c \in S_{2}-\{e\}$, then $a^{*}\left(b^{*} c\right)=a^{*} c=c^{*} a=c^{*}\left(a^{*} b\right)$;

(4) If $a \in S_{2}-\{e\}, b \in S_{2}-\{e\}$ and $c \in S_{1}-\{e\}$, then $a^{*}\left(b^{*} c\right)=a^{*} b=c^{*}\left(a^{*} b\right)$;

(5) If $a \in S_{1}-\{e\}, b \in S_{1}-\{e\}$ and $c \in S_{2}-\{e\}$, then $a^{*}\left(b^{*} c\right)=a^{*} c=c=c^{*}\left(a^{*} b\right)$;

(6) If $a \in S_{1}-\{e\}, b \in S_{2}-\{e\}$ and $c \in S_{1}-\{e\}$, then $a^{*}\left(b^{*} c\right)=a^{*} b=c^{*}\left(a^{*} b\right)$;

(7) If $a \in S_{2}-\{e\}, b \in S_{1}-\{e\}$ and $c \in S_{1}-\{e\}$, then $a^{*}\left(b^{*} c\right)=a=a^{*} b=c^{*}\left(a^{*} b\right)$.

Then $\left(S,{ }^{*}\right)$ is a variant CA-groupoid and $e$ is the quasi-right unit element.

Example 16. Let $S_{1}=\{1,2,3,4\}$ and $S_{2}=\{1,5,6,7\}$. Define operations ${ }^{*}$ and ${ }_{2}{ }_{2}$ on $S_{1}, S_{2}$ as following Tables 19 and 21. Then $S=S_{1} \cup S_{2}=\{1,2,3,4,5,6\}$, and $\left(S,{ }^{*}\right)$ is a variant $C A$-groupoid with the operation * in Table 20.

Table 19. The operation ${ }^{*}$ on $S_{1}$.

\begin{tabular}{lllll}
\hline${ }^{*} \mathbf{1}$ & $\mathbf{1}$ & $\mathbf{2}$ & $\mathbf{3}$ & $\mathbf{4}$ \\
\hline $\mathbf{1}$ & 2 & 2 & 4 & 4 \\
$\mathbf{2}$ & 2 & 2 & 4 & 4 \\
$\mathbf{3}$ & 3 & 4 & 4 & 4 \\
$\mathbf{4}$ & 4 & 4 & 4 & 4 \\
\hline
\end{tabular}


Table 20. The operation * on $S$.

\begin{tabular}{llllllll}
\hline $\boldsymbol{*}$ & $\mathbf{1}$ & $\mathbf{2}$ & $\mathbf{3}$ & $\mathbf{4}$ & $\mathbf{5}$ & $\mathbf{6}$ & $\mathbf{7}$ \\
\hline $\mathbf{1}$ & 2 & 2 & 4 & 4 & 5 & 6 & 7 \\
$\mathbf{2}$ & 2 & 2 & 4 & 4 & 5 & 6 & 7 \\
$\mathbf{3}$ & 3 & 4 & 4 & 4 & 5 & 6 & 7 \\
$\mathbf{4}$ & 4 & 4 & 4 & 4 & 5 & 6 & 7 \\
$\mathbf{5}$ & 5 & 5 & 5 & 5 & 5 & 7 & 7 \\
$\mathbf{6}$ & 6 & 6 & 6 & 6 & 7 & 5 & 5 \\
$\mathbf{7}$ & 7 & 7 & 7 & 7 & 7 & 5 & 5 \\
\hline
\end{tabular}

Table 21. The operation ${ }_{2}$ on $S_{2}$.

\begin{tabular}{lllll}
\hline $\boldsymbol{*}_{\mathbf{2}}$ & $\mathbf{1}$ & $\mathbf{5}$ & $\mathbf{6}$ & $\mathbf{7}$ \\
\hline $\mathbf{1}$ & 5 & 5 & 7 & 7 \\
$\mathbf{5}$ & 5 & 5 & 7 & 7 \\
$\mathbf{6}$ & 6 & 7 & 5 & 5 \\
$\mathbf{7}$ & 7 & 7 & 5 & 5 \\
\hline
\end{tabular}

Similar to Theorem 11, we can get another constructer method as following proposition (the proof is omitted).

Proposition 8. Let $\left(S_{1},{ }^{*}\right)$ and $\left(S_{2},{ }^{*}\right)$ be two variant $C A$-groupoids, $e_{1}$ and $e_{2}$ are variant unit elements of $\left(S_{1},{ }_{1}\right)$ and $\left(S_{2},{ }_{2}\right), S_{1} \cap S_{2}=\varnothing$ and $S_{2}$ is commutative. Denote $S=S_{1} \cup S_{2}$, and define the operation ${ }^{*}$ in $S$ as follows:

(1) if $a, b \in S_{1}$, then $a^{*} b=a^{*}{ }_{1}$;

(2) if $a, b \in S_{2}$, then $a^{*} b=a^{*}{ }_{2} b$;

(3) if $a \in S_{1}, b \in S_{2}$, then $a^{*} b=b$;

(4) if $a \in S_{2}, b \in S_{1}$, then $a^{*} b=a$.

Then $(S, *)$ is a variant $C A$-groupoid with the quasi-right unite $e_{1}$.

Example 17. Let $S_{1}=\{1,2,3,4\}$ and $S_{2}=\{5,6,7,8\}$. Define operations ${ }^{*}{ }_{1}$ and ${ }_{2}{ }_{2}$ on $S_{1}, S_{2}$ as following Tables 22 and 24. Then $S=S_{1} \cup S_{2}=\{1,2,3,4,5,6,7,8\}$, and $(S, *)$ is a variant $C A$-groupoid with the operation * in Table 23.

Table 22. The operation ${ }^{*}$ on $S_{1}$.

\begin{tabular}{lllll}
\hline${ }^{*} \mathbf{1}$ & $\mathbf{1}$ & $\mathbf{2}$ & $\mathbf{3}$ & $\mathbf{4}$ \\
\hline $\mathbf{1}$ & 3 & 2 & 2 & 4 \\
$\mathbf{2}$ & 2 & 2 & 2 & 4 \\
$\mathbf{3}$ & 3 & 2 & 2 & 4 \\
$\mathbf{4}$ & 4 & 4 & 4 & 4 \\
\hline
\end{tabular}


Table 23. The operation * on $S$.

\begin{tabular}{lllllllll}
\hline $\boldsymbol{*}$ & $\mathbf{1}$ & $\mathbf{2}$ & $\mathbf{3}$ & $\mathbf{4}$ & $\mathbf{5}$ & $\mathbf{6}$ & $\mathbf{7}$ & $\mathbf{8}$ \\
\hline $\mathbf{1}$ & 3 & 2 & 2 & 4 & 5 & 6 & 7 & 8 \\
$\mathbf{2}$ & 2 & 2 & 2 & 4 & 5 & 6 & 7 & 8 \\
$\mathbf{3}$ & 3 & 2 & 2 & 4 & 5 & 6 & 7 & 8 \\
$\mathbf{4}$ & 4 & 4 & 4 & 2 & 5 & 6 & 7 & 8 \\
$\mathbf{5}$ & 5 & 5 & 5 & 5 & 8 & 6 & 7 & 8 \\
$\mathbf{6}$ & 6 & 6 & 6 & 6 & 6 & 6 & 6 & 6 \\
$\mathbf{7}$ & 7 & 7 & 7 & 7 & 7 & 6 & 6 & 7 \\
8 & 8 & 8 & 8 & 8 & 8 & 6 & 7 & 8 \\
\hline
\end{tabular}

Table 24. The operation ${ }_{2}$ on $S_{2}$.

\begin{tabular}{lllll}
\hline $\boldsymbol{*}_{2}$ & 5 & 6 & 7 & 8 \\
\hline 5 & 8 & 6 & 7 & 8 \\
$\mathbf{6}$ & 6 & 6 & 6 & 6 \\
7 & 7 & 6 & 6 & 7 \\
8 & 8 & 6 & 7 & 8 \\
\hline
\end{tabular}

Theorem 12. Let $S_{1}$ be a variant $C A$-groupoid with order $n$ ( $n \geq 2$ and $n$ is an even number) and the quasi- right unit element $e_{1} \in S_{1}$, let $S_{2}$ be a variant $C A$-groupoid with order 2 and the quasi-right unit element $e_{2} \in S_{2}$. If $S$ $=S_{1} \cup S_{2}$ and $S_{1} \cap S_{2}=\varnothing$, then $S$ is a variant $C A$-groupoid, when it such that any of the following conditions:

(1) for the variant $C A$-groupoid $S$, the quasi-right unit element $e=e_{1}$, and $e_{2}^{*} e_{1}=e_{2}$, and for all $x \in S, x^{*}\left(e_{2}^{*}\right.$ $\left.e_{2}\right)=\left(e_{2}{ }^{*} e_{2}\right)^{*} x=e_{2}{ }^{*} e_{2}, x^{*} e_{2}=e_{2}{ }^{*} e_{2}, e_{2}{ }^{*} x=e_{2}{ }^{*} e_{2}\left(x \neq e_{1}\right)$;

(2) for the variant $C A$-groupoid $S$, the quasi-right unit element $e=e_{1}$ and for all $x \in S, x^{*}\left(e_{2}^{*} e_{2}\right)=\left(e_{2}^{*} e_{2}\right)^{*} x$ $=e_{2}{ }^{*} e_{2}, x^{*} e_{2}=e_{2}^{*} x=e_{2}$.

Proof. (1) Suppose that $S$ is constructed according to the method described in (1), then for all $x, y, z \in S_{1}$, $x^{*} y z=z^{*} x y=y^{*} z x$, and:

$$
\begin{gathered}
x^{*} y e_{2}=x^{*} e_{2} e_{2}=e_{2} e_{2}, e_{2} * x y=e_{2} e_{2}\left(x y \neq e_{1}\right) \\
y * e_{2} x=\left\{\begin{array}{c}
y * e_{2} e_{1}=y e_{2}=e_{2} e_{2} \quad x=e_{1} \\
y * e_{2} e_{2}=e_{2} e_{2} \quad x \neq e_{1}
\end{array}\right.
\end{gathered}
$$

That is, $x^{*} y e_{2}=e_{2}^{*} x y=y^{*} e_{2} x$. Denote $e_{2} e_{2}=b$, then:

$$
\begin{gathered}
x^{*} y b=x b=b, b^{*} x y=b, y^{*} b x=y b=b . \text { That is, } x^{*} y b=b^{*} x y=y^{*} b x . \\
x^{*} e_{2} e_{2}=x b=b, e_{2}^{*} x e_{2}=e_{2}^{*} e_{2} e_{2}=b, \\
e_{2} * e_{2} x=\left\{\begin{array}{cc}
e_{2} * e_{2} e_{1}=e_{2} e_{2}=b & x=e_{1} \\
e_{2} * e_{2} e_{2}=b & x \neq e_{1}
\end{array}\right.
\end{gathered}
$$

Thus, $x^{*} e_{2} e_{2}=e_{2}{ }^{*} x e_{2}=e_{2}{ }^{*} e_{2} x$. And:

$$
\begin{gathered}
x^{*} b e_{2}=x^{*} e_{2} b=x b=b, b^{*} x e_{2}=b^{*} e_{2} e_{2}=b=e_{2} b=e_{2}{ }^{*} b x, e_{2}{ }^{*} x b=e_{2} b=b, \\
\boldsymbol{b} * \boldsymbol{e}_{\mathbf{2}} \boldsymbol{x}=\left\{\begin{array}{c}
\boldsymbol{b} * \boldsymbol{e}_{\mathbf{2}} \boldsymbol{e}_{\mathbf{1}}=\boldsymbol{b}_{\mathbf{2}}=\boldsymbol{b} \quad \boldsymbol{x}=\boldsymbol{e}_{\mathbf{1}} \\
\boldsymbol{b} * \boldsymbol{e}_{\mathbf{2}} \boldsymbol{e}_{\mathbf{2}}=\boldsymbol{b} \quad \boldsymbol{x} \neq \boldsymbol{e}_{\mathbf{1}}
\end{array}\right.
\end{gathered}
$$

It follows that $x^{*} b e_{2}=e_{2} * x b=b^{*} e_{2} x$, and $x^{*} e_{2} b=b^{*} x e_{2}=e_{2}^{*} b x$. Obviously, $x^{*} b b=b^{*} x b=b^{*} b x$. Hence, $S$ is a variant CA-groupoid. 
(2) Suppose that $S$ is constructed according to the method described in (2), then for all $x, y, z \in S_{1}$, $x^{*} y z=z^{*} x y=y^{*} z x$, and:

$$
x^{*} y e_{2}=x e_{2}=e_{2}, e_{2}^{*} x y=e_{2}, y^{*} e_{2} x=y e_{2}=e_{2} .
$$

Then $x^{*} y e_{2}=e_{2}^{*} x y=y^{*} e_{2} x$. Assume $e_{2} e_{2}=b$, then:

$$
x^{*} y b=x b=b, b^{*} x y=b, y^{*} b x=y b=b .
$$

That is, $x^{*} y b=b^{*} x y=y^{*} b x$. And:

$$
x^{*} e_{2} e_{2}=x b=b, e_{2}^{*} x e_{2}=e_{2}{ }^{*} e_{2} x=e_{2} e_{2}=b .
$$

Thus, $x^{*} e_{2} e_{2}=e_{2}{ }^{*} x e_{2}=e_{2}{ }^{*} e_{2} x$. Moreover:

$$
x^{*} b e_{2}=x^{*} e_{2} b=x b=b, b^{*} x e_{2}=b e_{2}=b=e_{2} b=e_{2}^{*} b x, e_{2}^{*} x b=e_{2} b=b=b e_{2}=b^{*} e_{2} x .
$$

It follows that $x^{*} b e_{2}=e_{2}^{*} x b=b^{*} e_{2} x$, and $x^{*} e_{2} b=b^{*} x e_{2}=e_{2} * b x$. Obviously, $x^{*} b b=b^{*} x b=b^{*} b x$. Hence, $\mathrm{S}$ is a variant $\mathrm{CA}$-groupoid.

\section{Conclusions}

In the paper, we mainly study various cancellabilities of CA-groupoids and the structural properties of a special kind of CA-groupoids (variant CA-groupoids). Firstly, we investigate some cancellabilities of CA-groupoids, including left (right) cancellation, weak cancellation, left (right) quasi-cancellation and left (right) separation, and analyze the relationships among them. Secondly, from the view of quasi-right unit element, we introduce the new notion of variant CA-groupoid, illustrate the close connections among variant $\mathrm{CA}$-groupoid with commutative semigroup, ring and semiring by some examples; discuss deeply the characteristics of variant CA-groupoid, and establish its structure theorem and construction methods. This paper obtains many conclusions, some important results as follows:

(1) Every left cancellative element in CA-groupoid is right cancellative (see Theorem 1);

(2) For a CA-groupoid, it is left cancellative if and only if it is right cancellative (see Theorem 1 and Corollary 1);

(3) For a CA-groupoid, it is left separative if and only if it is right separative, and if and only if it is quasi-separative (see Theorem 4 and Corollary 1);

(4) Every left quasi-cancellative CA-groupoid is right quasi-cancellative (see Theorem 5); every power cancellative CA-groupoid is separative (see Theorem 7);

(5) For a variant CA-groupoid, its quasi-right unit element is unique;

(6) A variant CA-groupoid can be decomposed into the quasi-right unit element and a commutative CA-groupoid; starting from any commutative semigroup, one can construct a variant CA-groupoid (see Theorem 9);

(7) There are many ways to construct a new variant CA-groupoid from the existing variant CA-groupoids (see Theorems 11 and 12).

As a direction of future research, we will discuss the structural characteristics of CA-rings, CA-semirings and related algebraic systems (see [36-39]).

Author Contributions: X.Z., Z.M. initiated the research and wrote the paper, F.S. supervised the research work and provided helpful suggestions. All authors have read and agreed to the published version of the manuscript.

Funding: This research was funded by National Natural Science Foundation of China (Grant No. 61976130).

Conflicts of Interest: The authors declare no conflict of interest. 


\section{References}

1. Zhang, X.H.; Ma, Z.R.; Yuan, W.T. Cyclic associative groupoids (CA-groupoids) and cyclic associative neutrosophic extended triplet groupoids (CA-NET-groupoids). Neutrosophic Sets Syst. 2019, 29, 19-29.

2. Yuan, W.T.; Zhang, X.H. Regular CA-groupoids and cyclic associative neutrosophic extended triplet groupoids (CA-NET-groupoids) with green relations. Mathematics 2020, 8, 204. [CrossRef]

3. Byrne, L. Two brief formulations of Boolean algebra. Bull. Am. Math. Soc. 1946, 52, 269-272. [CrossRef]

4. Sholander, M. Medians, lattices, and trees. Proc. Am. Math. Soc. 1954, 5, 808-812. [CrossRef]

5. Hosszu, M. Some functional equations related with the associative law. Publ. Math. Debrecen. 1954, 3 , 205-214.

6. Maksa, G. CM solutions of some functional equations of associative type. Ann. Univ. Sci. Bp. Sect. Comput. 2004, 24, 125-132.

7. Kleinfeld, M. Rings with $\mathrm{x}(\mathrm{yz})=\mathrm{y}(\mathrm{zx})$. Commut. Algebra 1995, 23, 5085-5093. [CrossRef]

8. Behn, A.; Correa, I.; Hentzel, I.R. Semiprimality and nilpotency of nonassociative rings satisfying $x(y z)=y(z x)$. Commun. Algebra 2008, 36, 132-141. [CrossRef]

9. Behn, A.; Correa, I.; Hentzel, I.R. On flexible algebras satisfying $\mathrm{x}(\mathrm{yz})=\mathrm{y}(\mathrm{zx})$. Algebra Colloq. 2010, 17, 881-886. [CrossRef]

10. Iqbal, M.; Ahmad, I.; Shah, M.; Ali, M.I. On cyclic associative Abel-Grassman groupoids. Br. J. Math. Comput. Sci. 2016, 12, 1-16. [CrossRef]

11. Tamura, T. Commutative nonpotent archimedean semigroup with cancelation law I. J. Gakugei Tokushima Univ. 1957, 8, 5-11.

12. Burmistrovich, I.E. Commutative bands of cancellative semigroups. Sib. Mat. Zh. 1965, 6, 284-299.

13. Grillet, P.A. Cancellative commutative semigroups. Semigroup Forum. 1970, 1, 249-253. [CrossRef]

14. Neumann, B.H. Some remarks on cancellative semigroups. Math. Z. 1970, 117, 97-111. [CrossRef]

15. Goodearl, K.R. Power-cancellation of groups and modules. Pac. J. Math. 1976, 64, 387-411. [CrossRef]

16. Cherubini, A.; Varisco, A. Power cancellative semigroups. Semigroup Forum. 1979, 18, 381-384.

17. Lezzi, D. The Para-cancellation law in commutative semigroups. Acta Math. Acad. Sci. Hung. Tomus. 1980, 36, 65-69.

18. Krasilnikova, Y.I.; Novikov, B.V. On quasi-separative semigroups. Semigroup Forum 2005, 70, 347-355. [CrossRef]

19. Cegarra, A.M.; Petrich, M. The rank of a commutative cancellative semigroup. Acta Math. Hungar. 2005, 107, 71-75.

20. Cegarra, A.M.; Petrich, M. Commutative cancellative semigroups of low rank. Results Math. 2009, 54, 41-52. [CrossRef]

21. Shah, M.; Ali, A. Some structure properties of AG-groups. Int. Math. Forum. 2011, 6, 1661-1667.

22. Ali, A.; Shah, M.; Ahmad, I. On quasi-cancellativity of AG-groupoids. Int. J. Contemp. Math. Sci. 2012, 7, 2065-2070.

23. Shah, M.; Shah, T.; Ali, A. On the cancellativity of AG-groupoids. Int. Math. Forum. 2011, 6, 2187-2194.

24. Petrich, M. Malcev products of weakly cancellative monoids and varieties of bands. Semigroup Forum. 2015, 90, 339-373. [CrossRef]

25. Zhang, X.H.; Smarandache, F.; Liang, X.L. Neutrosophic duplet semigroup and cancellable neutrosophic triplet groups. Symmetry 2017, 9, 275. [CrossRef]

26. Zhang, X.H.; Ma, Y.C.; Yu, P. On two conjectures of Abel Grassmann's groupoids. Symmetry 2019, 11, 816. [CrossRef]

27. Steinberg, B. Representation Theory of Finite Monoids; Springer: Berlin/Heidelberg, Germany, 2016.

28. Protic, P. Some remarks on Abel-Grassmann's groups. Quasigroups Relat. Syst. 2012, 20, 267-274.

29. Shah, M. A Theoretical and Computational Investigation of AG-groups. Ph.D Thesis, Quaidi-Azam University Islamabad, Islamabad, Pakistan, 2012.

30. Stanovsky, D. Linear representation of Abel-Grassmann groups. Carpathian J. Math. 2017, 33, $257-263$.

31. Zhang, X.H.; Wu, X.Y. Involution Abel-Grassmann's groups and filter theory of Abel-Grassmann's groups. Symmetry 2019, 11, 553. [CrossRef]

32. Zhang, X.H.; Wu, X.Y.; Mao, X.Y. On neutrosophic extended triplet groups (loops) and Abel-Grassmann's groupoids (AG-groupoids). J. Intell. Fuzzy Syst. 2019, 37, 5743-5753. [CrossRef] 
33. Golan, J.S. Semirings and Their Applications; Springer: Dordrecht, The Netherlands, 1999.

34. Gondran, M.; Minoux, M. Dioïds and semirings: Links to fuzzy sets and other applications. Fuzzy Sets Syst. 2007, 158, 1273-1294. [CrossRef]

35. Zumbragel, J. Classification of finite congruence-simple semirings with zero. J. Algebra Appl. 2008, 7, 363-377. [CrossRef]

36. Zhang, X.H.; Borzooei, R.A.; Jun, Y.B. Q-filters of quantum B-algebras and basic implication algebras. Symmetry 2018, 10, 573. [CrossRef]

37. Zhang, X.H.; Hu, Q.Q.; Smarandache, F.; An, X.G. On neutrosophic triplet groups: Basic properties, NT-subgroups, and some notes. Symmetry 2018, 10, 289. [CrossRef]

38. Wu, X.Y.; Zhang, X.H. The decomposition theorems of AG-neutrosophic extended triplet loops and strong AG-(1, 1)-loops. Mathematics 2019, 7, 268. [CrossRef]

39. Zhang, X.H.; Wang, X.J.; Smarandache, F.; Jaiyeola, T.G.; Lian, T.Y. Singular neutrosophic extended triplet groups and generalized groups. Cogn. Syst. Res. 2019, 57, 32-40. [CrossRef]

(C) 2020 by the authors. Licensee MDPI, Basel, Switzerland. This article is an open access article distributed under the terms and conditions of the Creative Commons Attribution (CC BY) license (http://creativecommons.org/licenses/by/4.0/). 